\title{
Переход между электронной локализацией и антилокализацией, а также проявление фазы Берри в графене на поверхности SiC
}

\author{
(C) Н.В. Агринская ${ }^{1}$, А.А. Лебедев ${ }^{1}$, С.П. Лебедев ${ }^{3}$, M.A. Шахов ${ }^{1}$, E. Lahderanta ${ }^{2}$ \\ ${ }^{1}$ Физико-технический институт им. А.Ф. Иоффе Российской академии наук, \\ 194021 Санкт-Петербург, Россия \\ ${ }^{2}$ Department of Mathematics and Physics, Lappeenranta University of Technology, \\ Lappeenranta, Finland \\ ${ }^{3}$ Университет ИТМО, \\ 197101 Санкт-Петербург, Россия \\ E-mail: nina.agrins@mail.ioffe.ru
}

(Получена 17 апреля 2018 г. Принята к печати 27 мая 2018 г.)

Показано, что транспортные свойства графитизированного карбида кремния определяются слоем графена на поверхности $\mathrm{SiC}$, сильно легированным электронами. В слабых магнитных полях и при низких температурах наблюдалось отрицательное магнетосопротивление, являющееся следствием слабой локализации. Впервые в таких образцах в магнетосопротивлении при повышении температуры наблюдался переход от слабой локализации к слабой антилокализации (последняя является проявлением изоспина в графене). В сильных магнитных полях (до 30 Тл) наблюдалась выраженная картина осцилляций Шубникова-де Гааза, которая демонстрирует 4-кратное вырождение спектра носителей вследствие двойного спинового и двойного долинного вырождений, а также проявление фазы Берри. Оценена эффективная масса электронов $m^{*}=0.08 m_{0}$, которая характерна для графена с высокой концентрацией носителей.

DOI: $10.21883 /$ FTP.2018.12.46767.8892

\section{1. Введение}

В настоящее время графен (представляющий собой изолированный одноатомный слой графита) рассматривается как один из самых перспективных двумерных материалов для применения в наноэлектронике. Однако свойства графена существенно отличаются от хорошо изученных двумерных квантовых ям в полупроводниках. К таким свойствам относятся полуцелочисленный квантовый холл-эффект и существование ненулевой фазы Берри, что является спецификой дираковских фермионов или псевдоспина. Эти отличия связаны с уникальной топологией зонной структуры графена. Кроме чисто научного интереса, эти уникальные квантовые транспортные явления могут привести к новым применениям графена в магнетоэлектронике [1].

Несмотря на то что графен в настоящее время является достаточно хорошо изученным материалом, это в основном относится к монослоям графена, механически нанесенного на подложку. Что касается графена, выращенного на подложке, здесь понимания значительно меньше. В этом случае свойства графена зависят от конкретной подложки и характера связи с подложкой. Для механически нанесенного монослоя графена предполагается наличие квантовых поправок к проводимости, вызванных эффектом слабой антилокализации (WAL), связанным с проявлением фазы Берри. Однако для графена, выращенного на подложках, в магнетосопротивлении при низких температурах наблюдаются эффекты слабой локализации (WL). В работе [2] это объяснялось тем, что время междолинных переходов за- метно меньше, чем время дефазировки. Такое поведение ожидается для графена, сильно связанного с подложкой, при этом пространственный масштаб рассеивателей на подложке сравним с атомным, т.е. на больших траекториях в разупорядоченном графене псевдоспин может не проявляться [3]. Однако в эффектах, которые связаны с малыми траекториями (циклотронная орбита для осцилляций Шубникова-де Гааза), псевдоспин и фаза Берри должны проявляться.

Наиболее перспективной технологией синтеза графена, которая позволяет получать высококачественный материал достаточно больших размеров и в то же время может быть интегрирована в промышленное производство, представляется термодеструкция поверхности полуизолирующих подложек карбида кремния ( $\mathrm{SiC})$. Такие структуры также достаточно хорошо изучены [4-6], однако полного понимания влияния интерфейсного слоя не существует. В частности, это связано с тем, что вследствие дефектного характера интерфейса подвижность носителей в таких структурах существенно меньше, чем в механически нанесенных монослоях графена. В таких образцах некоторые квантовые эффекты, такие как осцилляции Шубникова-деГааза (ШдГ), обычно проявляются слабо [7].

В данной работе будут представлены результаты изучения квантовых транспортных свойств графена, выращенного методом термодеструкции на поверхности $\mathrm{SiC}(0001)$ в среде аргона, как в слабых, так и в сильных магнитных полях, до 30 Тл. В частности, мы наблюдали очень четкую картину осцилляций ШдГ и установили наличие аномальной фазы Берри. Кроме того, впервые 
наблюдался переход от слабой локализации к слабой антилокализации в магнетосопротивлении слоя графена при повышении температуры до $200 \mathrm{~K}$.

\section{2. Эксперимент}

Для подготовки пленок графена к проведению электрофизических измерений на поверхности образцов формировались тестовые структуры в геометрии холловского моста (Hall-bar). Топология требуемых структур была создана с применением стандартной процедуры процесса контактной фотолитографии с использованием специально разработанного комплекта фотошаблонов. В области температур $4-300 \mathrm{~K}$ и магнитных полей 0-35 Тл были измерены эффект Холла, проводимость и магнетосопротивление. По знаку холловского сигнала установлен электронный тип носителей. Концентрация электронов, определенная из эффекта Холла при низких магнитных полях, составляла $1.2 \cdot 10^{13} \mathrm{~cm}^{-2}$ и очень слабо менялась с температурой в диапазоне температур от 300 до $4 \mathrm{~K}$. Такая большая концентрация связана с собственными дефектами на интерфейсе и означает, что химический потенциал лежит примерно на 420 мэВ выше дираковской точки [6]. В соответствии с данными эффекта Холла подвижность носителей составляла $300-400 \mathrm{~cm}^{2} / \mathrm{B} \cdot \mathrm{c}$ в диапазоне температур $300-4 \mathrm{~K}$.

Температурная зависимость проводимости представлена на рис. 1. Начиная с высоких температур, проводимость растет (сопротивление падает) с уменьшением температуры, $R \propto T$ (см. вставку на рис. 1 ), что соответствует металлическому поведению двумерных носителей заряда при рассеянии на акустических фононах [8]. Далее проводимость начинает падать с уменьшением температуры в интервале $30-2 \mathrm{~K}$, при этом зависимость проводимости от $T$ четко следует логарифмическому закону в соответствии с поведением, ожидаемым для

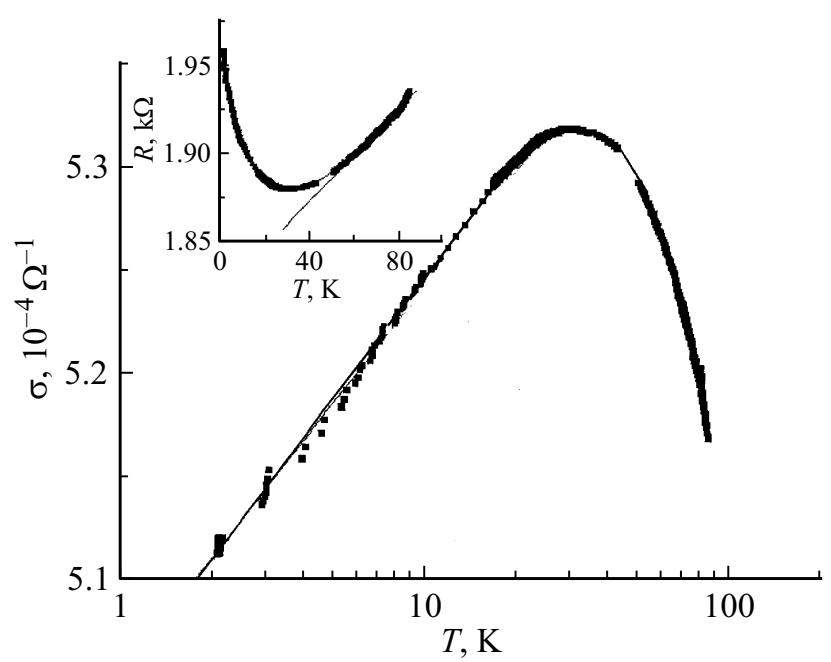

Рис. 1. Температурная зависимость проводимости при $B=0$. На вставке - высокотемпературная часть зависимости сопротивления.

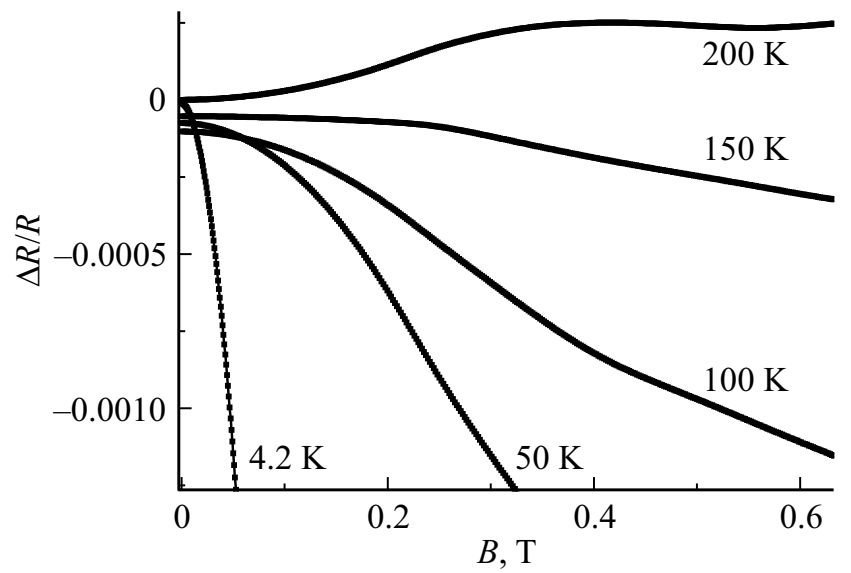

Рис. 2. Кривые магнетосопротивления в слабых магнитных полях при различных температурах.

слабой локализации $-\mathrm{WL}$ (т.е. интерференционной поправки в двумерную проводимость). Для графена интерференционная поправка равна (см., например, [3])

$$
\Delta \sigma(T) \propto-\left(e^{2} / \pi h\right) \ln \left(\tau_{\varphi} / \tau_{t r}\right),
$$

где $\tau_{\varphi} \propto T^{-1}-$ время сбоя фазы, $\tau_{t r}-$ время релаксации импульса в графене. Наклон зависимости на рис. 1 дает значение $0.2 \cdot 10^{-4} \mathrm{OM}^{-1}$ или, в терминах шкалы натуральных логарифмов, $\sim 0.8 \cdot 10^{-5} \mathrm{OM}^{-1}$, что соответствует коэффициенту в выражении (1) .

На рис. 2 показаны зависимости магнетосопротивления (магнитное поле перпендикулярно плоскости образца) при различных температурах.

В области слабых магнитных полей $(B<1$ Тл) и низких температур был обнаружен эффект отрицательного магнетосопротивления (ОМC), величина которого уменьшалась с ростом температуры 100-4K. Этот эффект имеет интерференционную природу и связан с WL. Более подробное объяснение будет дано в разд. 3. С другой стороны, в области высоких температур (при $T=150-200 \mathrm{~K})$ в слабых магнитных полях $\sim 0.2$ Тл наблюдался сравнительно резкий рост положительного магнетосопротивления (ПМС), тогда как дальнейший рост поля приводит к переходу к классическому квадратичному ПМС. Мы рассматриваем начальный рост ПМС как проявление слабой антилокализации (WAL), характерной для графена, вследствие свойств носителей заряда (наличие псевдоспина, приводящего к проявлению фазы Берри). Таким образом, с ростом температуры мы наблюдали переход от WL к WAL. При дальнейшем повышении температуры до $250 \mathrm{~K}$ интерференционные квантовые эффекты исчезают и остается классическое ПМС для свободных электронов.

Далее, было измерено магнетосопротивление и эффект Холла при $T=4.2 \mathrm{~K}$ в сильных магнитных полях. На кривых $R_{x y}(B)$ не было обнаружено проявления квантового эффекта Холла, на кривых $R_{x x}(B)$ наблюдались четко выраженные осцилляции ШдГ (рис. 3). 


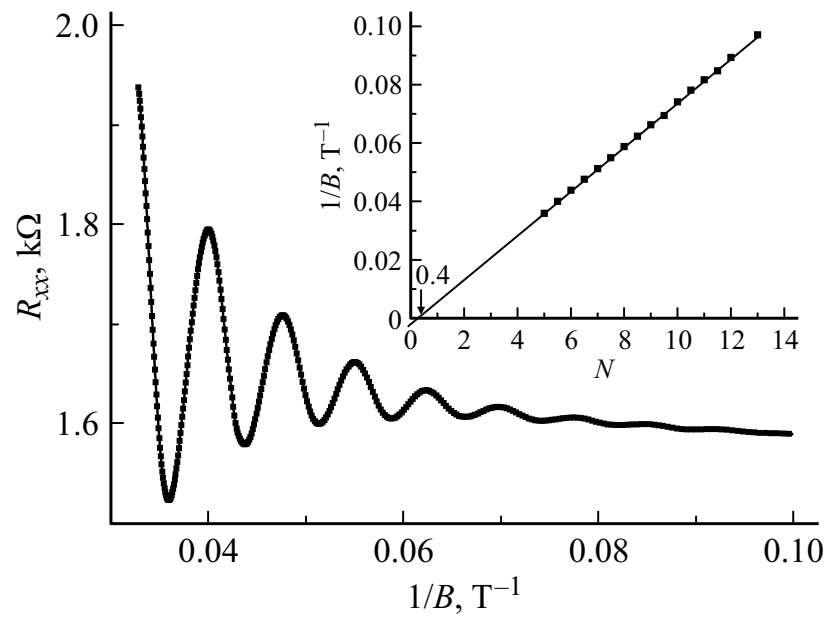

Рис. 3. Кривая магнетосопротивления в масштабе обратного магнитного поля, полученная вычетом фонового МС (полином 2-го порядка). На вставке - номера уровней Ландау как функция положения максимумов (полуцелые номера) и минимумов (целые номера) для осцилляций ШдГ. Для графена отсечка по горизонтальной оси должна быть $\beta / 2 \pi=0.5$, если фаза Берри равна $\beta=\pi$.

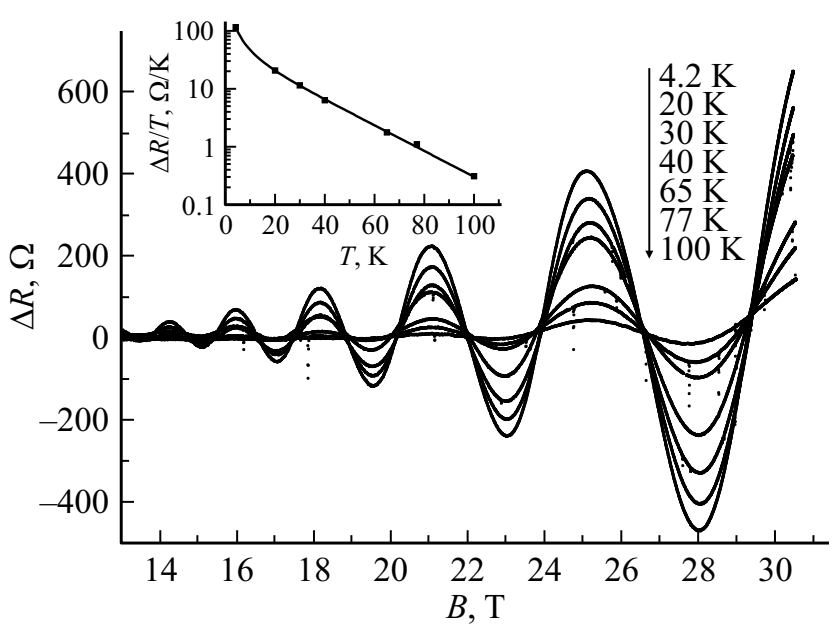

Рис. 4. Температурная зависимость амплитуды осцилляций ШдГ - $A(T, B)$. На вставке - для поля 28 Тл: точки - эксперимент, кривая - построена в соответствии с выражением (2).

На вставке к рис. 3 показаны положения максимумов (полуцелые числа уровней Ландау) и минимумов (целые числа) осцилляций ШдГ в масштабе $1 / B$. Видно, что эта зависимость прямолинейна с наклоном $0.00745 / B$ и по оси ординат экстраполируется в $N=(0.4-0.35)$. Для графена отсечка по горизонтальной оси должна быть $\beta / 2 \pi=0.5$, если фаза Берри равна $\beta=\pi$. Это означает фазовый сдвиг порядка $\pi$ по отношению к стандартному квантованию Ландау в металлах. Этот сдвиг наблюдается в моноатомных пленках графена и связан с фазой Берри (зависимость чисел уровней Ландау от $1 / B$ экстраполируется в 0.5). В пленках графита из-за отсутствия фазы Берри зависимость чисел уровней Ландау от $1 / B$ экстраполируется в 0 [9].

С другой стороны, частота осцилляций ШдГ $\Delta=\phi / f \cdot n$, где $\phi=4.14 \cdot 10^{-11} \mathrm{Tл} \cdot \mathrm{cm}^{-2}$ - квант магнитного потока, $f-$ фактор вырождения, $n-$ концентрация носителей. Для графена $f=4$ вследствие двойного спинового и двойного долинного вырождения. Вычисление концентрации носителей из частоты дает значение $1.3 \cdot 10^{13} \mathrm{~cm}^{-2}$, что хорошо совпадает с холловскими данными для $4.2 \mathrm{~K}$. Поскольку осцилляции были хорошо видны до $100-150 \mathrm{~K}$, была построена температурная зависимость амплитуды $A(T, B)$ (см. рис. 4):

$$
\begin{gathered}
A(T, B) / T=\left(2 \mu \pi^{2} k_{B} / h \omega_{c}\right) \cdot\left(1 / \sin h\left(2 \pi^{2} k_{B} T / h \omega_{c}\right),\right. \\
\omega_{c}=e B / m c .
\end{gathered}
$$

Кривая хорошо ложится на экспериментальные точки, значение приведенной массы для электронов $m^{*}=0.08 m_{0}$. Для графена эффективная масса электронов сильно растет вблизи дираковской точки с ростом концентрации носителей.

$$
m=E_{F} / v_{F}=\left(\pi h^{2} n / v_{F}^{2}\right)^{1 / 2}
$$

где $n-$ концентрация носителей, $v_{F}=1.1 \cdot 10^{8} \mathrm{~cm} / \mathrm{c}$. Для $n=1 \cdot 10^{13} \mathrm{~cm}^{-2}$ получается $m^{*}=0.07 m_{0}$, что неплохо согласуется с нашей оценкой.

Несмотря на низкую подвижность, можно четко наблюдать порядка 10 осцилляций и построить зависимость амплитуды осцилляций от ее номера или график Дингла при $T=4.2 \mathrm{~K}$ (рис. 5).

$$
\begin{gathered}
\ln \left[\left(A\left(T, B_{n}\right) \cdot B_{n}^{-1 / 2} \cdot \sin h \chi\right) / \chi\right]=C-\pi m^{*} e \tau_{q}\left(1 / B_{n}\right), \\
\chi=2 \pi^{2} k_{\mathrm{B}} T / h \omega_{c} .
\end{gathered}
$$

Отсюда, зная эффективную массу графена для данной концентрации $m^{*}=0.08 m_{0}$, можно оценить $\tau_{q}$ квантовое время жизни $\simeq 14.5 \cdot 10^{-15}$ с. Линейная зависимость показывает, что это время не зависит от магнитного поля. Полученное таким образом квантовое

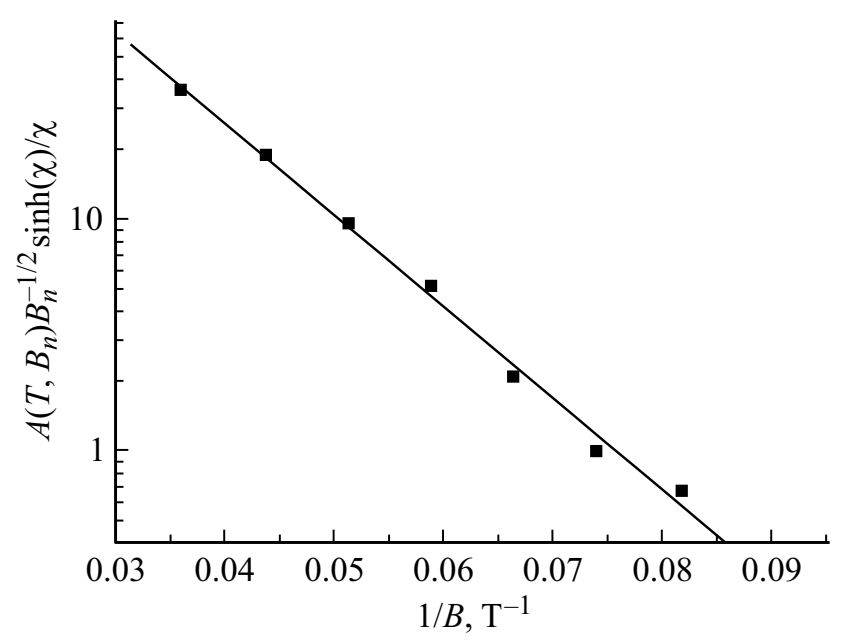

Рис. 5. Зависимость амплитуды осцилляций от ее номера при $T=4.2 \mathrm{~K}$. 
время жизни можно сравнить с транспортным временем $\tau_{t}$, полученным из значений холловской подвижности $\mu_{h}=400 \mathrm{~cm}^{2} / \mathrm{B} \cdot$ с при $T=4.2 \mathrm{~K}$. Вычисленное значение $\tau_{t}=\mu_{h} m^{*} / e \simeq 20 \cdot 10^{-15} \mathrm{c}$, отношение $\tau_{t} / \tau_{q}=1.4$. Превышение значения $\tau_{t}$ над $\tau_{q}$ является естественным, поскольку квантовое (,уходное“) время релаксации всегда меньше, чем транспортное (поскольку учитывает рассеяние на любые углы, тогда как транспортное учитывает лишь рассеяние на достаточно большие углы, приводящее к релаксации импульса). Таким образом, низкотемпературные данные в высоких магнитных полях: наличие фазы Берри (необычным является ее отклонение от стандартной величины в меньшую сторону) и 4-кратное вырождение спектра носителей, а также значение приведенной массы для электронов $m^{*}=0.08 m_{0}$ подтверждают, что проводимость соответствует проводимости однослойного графена. Данные эффекты характерны для графена и слабо чувствительны к дефектам на подложке [10].

\section{3. Обсуждение результатов}

Как было показано в данных образцах графена, на $\mathrm{SiC}$ наблюдаются хорошо выраженные осцилляции ШдГ, несмотря на низкие значения подвижности и высокие концентрации носителей. Это связано с применением высоких магнитных полей до 30 Тл. Как легко оценить, значение ларморовской частоты в полях 30 Тл для эффективной массы $0.08 m_{0}$ составляет $10^{14} \mathrm{c}^{-1}$, так что интервал между уровнями Ландау составляет примерно 70 мэВ. Соответственно, учитывая, что положение уровня Ферми для концентрации $1 \cdot 10^{13} \mathrm{~cm}^{-2}$ порядка 400 мэВ [6], число наблюдаемых осцилляций составляет порядка 6, что согласуется с экспериментом. С другой стороны, циклотронный период при этом может оказаться недостаточно малым по сравнению с временем междолинного рассеяния (время релаксации, определенное из графиков Дингла, составляет $1.3 \cdot 10^{-14} \mathrm{c}$ ), чтобы обеспечить идеальную проработку фазы Берри. Последнее обстоятельство может объяснять отличие наблюдаемого значения фазы Берри от „идеального“ значения $\pi$, которое бы обеспечило отсечку на вставке к рис. 3, равную 0.5 .

Далее было показано, что в данных образцах наблюдается переход от отрицательного магнетосопротивления (слабая локализация) к положительному (слабая антилокализация) с ростом температуры. Как известно, в слабых полях интерференционная поправка к магнетопроводимости в графене, возникающая в результате разложения общего выражения для проводимости по магнитному полю, может быть оценена как (см., напримep, [3])

$$
\begin{aligned}
\Delta \sigma(B) & =\left(e^{2} / 24 \pi h\right)\left(4 e D B \tau_{\varphi} / \mathrm{ch}\right)^{2} \\
& \times\left[1-1 /\left(1+2 \tau_{\varphi} / \tau_{i}\right)^{2}-2 /\left(1+\tau_{\varphi} / \tau_{i}+\tau_{\varphi} / \tau_{0}\right)^{2}\right],
\end{aligned}
$$

где $D-$ коэффициент диффузии, $\tau_{\varphi}-$ время сбоя фазы, $\tau_{i}$ и $\tau_{0}-$ характерные времена междолинной и внутридолинной релаксации соответственно. Прежде всего заметим, что при $\tau_{\varphi}<\tau_{i}$ фактор

$$
\left(4 e D B \tau_{\varphi} / \mathrm{ch}\right)^{2}
$$

в уравнении [3] описывает квадратичное по полю ОМС (отношение магнитного потока, пронизывающего контур с масштабом длины дефазировки $D \tau_{\varphi}$ и в области слабых полей, полагается малым). В области более сильных полей вместо этого, квадратичного по полю, фактора возникает логарифмический. Таким образом, мы можем грубо оценить время $\tau_{\varphi}$, оценив (для данной температуры) значение поля, при котором указанный фактор сравнивается с единицей. Из рис. 2 соответствующие значения полей можно оценить как $1.6 \cdot 10^{-1}$ Тл $(T=100 \mathrm{~K}), \quad 0.8 \cdot 10^{-1}$ Тл $(T=50 \mathrm{~K})$ и $0.7 \cdot 10^{-2}$ Тл $(T=4.2 \mathrm{~K})$. Имея в виду, что $D=\tau_{t} v_{F}^{2} / 2$, и подставляя соответствующие значения для $\tau_{t} \sim 2 \cdot 10^{-14} \mathrm{c}$, $v_{F} \sim 10^{8} \mathrm{~cm} / \mathrm{c}$, получаем для $4.2 \mathrm{~K} \tau_{\varphi} \sim 0.5 \cdot 10^{-11}$ с, для $50 \mathrm{~K} \tau_{\varphi} \sim 0.6 \cdot 10^{-12}$ с, тогда как для температур $100 \mathrm{~K}$ $\tau_{\varphi} \sim 0.3 \cdot 10^{-12} \mathrm{c}$; изменение $\tau_{\varphi}(T)$ хорошо описывается законом $\propto T^{-1}$, ожидаемым для электрон-электронного рассеяния. Эти обстоятельства позволяют сделать вывод о том, что наблюдаемое поведение $\Delta \sigma(B)$, так же как и поведение $\Delta \sigma(T)$, действительно связано с интерференционными эффектами.

В свою очередь фактор второго члена в квадратных скобках в уравнении (5) определяется соотношением между $\tau_{\varphi}$ и $\tau_{i}$. В частности, для достаточно больших температур этот фактор - независимо от величины магнитного поля - приводит к ПМС или к слабой антилокализации. Таким образом, поведение образца в слабых полях определяется соотношением междолинного рассеяния и рассеянием с потерей фазы, тогда как последнее чувствительно к температуре. Поскольку наблюдения квантовых поправок в слабых полях возможно лишь при достаточно низкой температуре, достаточно типичным обычно полагается переход от слабой локализации к слабой антилокализации с ростом поля [7]. Действительно, с ростом поля становятся актуальными все более короткие траектории, и междолинное рассеяние (проявляющееся в области слабых полей) перестает быть актуальным. Мы имеем дело с несколько иной ситуацией, когда поля являются существенно более сильными, чем в экспериментах [7]. Во всяком случае, в области сравнительно малых температур, когда длина дефазировки велика, во всей наблюдаемой области полей мы имеем дело лишь со слабой локализацией (вклад очень малых траекторий является пренебрежимо малым). В то же время в области сильных полей начинает проявляться чисто классический, квадратичный по полю механизм, о котором мы писали выше. Что же касается области высоких температур (которые, как правило, не рассматривались в предыдущих экспериментах в силу недостаточной величины использовавшихся полей), то, 
как мы видим, в области слабых полей обнаруживается достаточно заметный и сравнительно резкий рост положительного магнетосопротивления, который мы связываем с проявлением антилокализации на сравнительно коротких траекториях (обусловленных малостью длины сбоя фазы при больших температурах). Затем следует сравнительно плавный рост, обусловленный классическим магнетосопротивлением. Поэтому мы подчеркиваем, что в наших экспериментах мы наблюдаем кроссовер от слабой локализации к слабой антилокализации не с ростом магнитного поля (как в прежних экспериментах [7] ), а с ростом температуры. А именно в области малых температур в слабых полях наблюдается слабая локализация, а в области высоких температур - слабая антилокализация, тогда как дальнейший рост поля в обоих случаях приводит к переходу к классическому магнетосопротивлению.

С целью качественного обсуждения этого перехода отметим, что если грубо оценивать классическую магнетопроводимость как $(\Delta \sigma)_{c l} \sim-\sigma(\Omega \tau)^{2}$, где $\Omega-$ ларморовская частота, $\tau$ - транспортное время релаксации, то отношение интерференционной поправки $(\Delta \sigma)_{\text {int }}$ к классической можно переписать в виде

$$
(\Delta \sigma)_{\mathrm{int}} /(\Delta \sigma)_{c l} \sim C\left(\left(e^{2} / h\right) / \sigma\right)\left(\varepsilon \tau_{\varphi} / h\right) \cdot[A],
$$

где $\varepsilon-$ энергия, $C-$ некоторый численный коэффициент, $A$ зависит от соотношения между соответствующими временами релаксации. Можно усмотреть, что знак интерференционной поправки зависит от знака $A$, причем при больших временах $\tau_{\varphi}$ (по сравнению с $\tau_{i}$ ) знак $A$ отрицательный (слабая локализация), тогда как с ростом температуры, когда $\tau_{\varphi}$ резко уменышается, знак меняется - антилокализация. Качественно, антилокализационное поведение обусловлено тем, что характерное время движения по замкнутой траектории, ответственной за интерференционное поведение (ограниченное значением $\left.\tau_{\varphi}\right)$, в соответствующей ситуации оказывается меньше времени междолинных переходов. Соответственно для таких траекторий фаза Берри проявляется в полной мере, и антилокализационное поведение, типичное для изолированных слоев графена, восстанавливается. При этом в соответствующей области магнитных полей, где справедливо выражение (5), как видно из (6), интерференционная поправка может превосходить классическое магнетосопротивление (в силу большого значения параметра) $\varepsilon \tau_{\varphi} / h$, если отношение $\left(e^{2} / h\right) / \sigma$ не слишком мало. Однако с ростом поля в условиях, когда параметр

$$
4 \Omega \tau\left(\varepsilon \tau_{\varphi}\right) / h
$$

становится больше единицы, зависимость интерференционной поправки от магнитного поля становится значительно более слабой (логарифмической), тогда как классический эффект сохраняет квадратичную зависимость от поля. В результате при более сильных полях классический вклад становится преобладающим (и таким образом квадратичным по магнитному полю). Так что итоговая зависимость проводимости от поля должна быть квадратичной в слабых и сильных полях, обнаруживая участок более слабой зависимости от поля.

\section{4. Заключение}

Таким образом, графен, полученный на поверхности $\mathrm{SiC}$ методом термодеструкции, обнаруживает ряд несомненных свойств, характерных для графена. Наблюдается выраженная картина осцилляций ЩдГ в сильных полях, которая показывает 4-кратное вырождение спектра носителей вследствие двойного спинового и двойного долинного вырождения. Кроме того, было установлено наличие фазы аномальной Берри, ее отличие от „идеального“ значения $\pi$. Оцененное из температурной зависимости амплитуды осцилляций ШдГ значение приведенной массы для данных сильно легированных образцов графена составляет $m^{*}=0.08 m_{0}$. Впервые для данных структур в кривых магнетосопротивления наблюдался переход от слабой локализации к слабой антилокализации (последняя является подписью изоспина в графене) при повышении температуры до $200 \mathrm{~K}$. Интересно, что в графене на $\mathrm{SiC}$ эффект слабой антилокализации наблюдается при достаточно высоких температурах вследствие слабого электрон-фононного рассеяния [2].

Авторы признательны В.И. Козубу за прочтение и полезные обсуждения рукописи.

\section{Список литературы}

[1] Y. Zhang, Y.-W. Tan, H.L. Stormer, P. Kim. Nature Lett., 438, 201 (2005).

[2] F.V. Tikhonenko, A.A. Kozikov, A.K. Savchenko, R.V. Gorbachev. Phys. Rev. Lett., 103, 226801 (2009).

[3] E. Mc Cann, K. Kechedzhi, V.I. Fal'ko, H. Suzuura, T. Ando, B.L. Altshuler. Phys. Rev. Lett., 97, 146805 (2006).

[4] E. Tiras, S. Ardali, T. Tiras, E. Arslan, S. Cakmakyapan, O. Kazar, J. Hassan, E. Janzben, E. Ozbay. J. Appl. Phys., 113, 043708 (2013).

[5] E. Arslan, S. Ardali, E. Tiras, S. Cakmakyapan, E. Ozbay. Phil. Mag., 97 (3), 187 (2017).

[6] C. Reidl, C. Coletti, U. Starke. J. Phys. D: Appl. Phys., 43, 374009 (2010).

[7] X. Wu, X. Li, Z. Song, C. Berger, W.A. de Heer. Phys. Rev. Lett., 98, 136801 (2007).

[8] В. Карпус. ФТП, 20, 12 (1986).

[9] K.S. Novoselov, A.K. Geim1, S.V. Morozov, D. Jiang, M.I. Katsnelson, I.V. Grigorieva, S.V. Dubonos, A.A. Firsov. Nature Lett., 438, 197 (2005).

[10] K. Bennaceur, J. Guillemette, P.L. Lievesque, N. Cottenye, F. Mahvash, N. Hemsworth, A. Kumar, Y. Murata, S. Heun, M.O. Goerbig, C. Proust, M. Siaj, R. Martel, G. Gervais, T. Szkopek. Phys. Rev. B, 92, 125410 (2015).

Редактор Г.А. Оганесян 


\section{Transition between electron localization and antilocalization as well as manifestation of Berry's phase in graphene on the surface of SiC}

N.V. Agrinskaya ${ }^{1}$, A.A. Lebedev ${ }^{1}$, S.P. Lebedev ${ }^{3}$, M.A. Shakhov ${ }^{1}$, E. Lahderanta ${ }^{2}$

${ }^{1}$ loffe Institute, 194021 St. Petersburg, Russia

${ }^{2}$ Department of Mathematics and Physics, Lappeenranta University of Technology,

Lappeenranta, Finland

${ }^{3}$ University ITMO,

197101 St. Petersburg, Russia

Abstract It is shown that transport properties of graphitized silicon carbide are controlled by surface graphene layer strongly doped by electrons. At weak magnetic fields and low temperatures a negative magnetoresistance being a consequence of weak localization was observed. We present a first observation in these samples of a crossover in magnetoresistance from weak localization to weak antilocalization (the latter is a manifestation of isospin in graphene). The pronounced picture of Shubnikov-deHaas oscillations was observed at strong magnetic fields (up to $30 \mathrm{~T}$ ) which demonstrated 4-fold degeneration of carrier spectrum due to double spin degeneration and double valley degeneration. We also observed a manifestation of the Berry phase. The electron effective mass was estimated to be $m^{*}=0.08 m_{0}$ which is characteristic for graphene with high carrier concentration. 